\title{
Diabetes-associated microbiota in fa/fa rats is modified by Roux-en-Y gastric bypass
}

\author{
Tulika Arora ${ }^{1,7}$, Florian Seyfried ${ }^{2,3}$, Neil G Docherty ${ }^{4,5}$, Valentina Tremaroli ${ }^{1}$, \\ Carel W le Roux ${ }^{2,4,5}$, Rosie Perkins ${ }^{1}$ and Fredrik Bäckhed ${ }^{1,6}$ \\ ${ }^{1}$ Wallenberg Laboratory, Sahlgrenska Center for Cardiovascular and Metabolic Research, Department of \\ Molecular and Clinical Medicine, Institute of Medicine, University of Gothenburg, Gothenburg, Sweden; \\ ${ }^{2}$ Department of Investigative Medicine, Imperial College London, London, UK; ${ }^{3}$ Department of General, \\ Visceral, Vascular and Pediatric Surgery, University Hospital of Wuerzburg, Wuerzburg, Germany; ${ }^{4}$ Diabetes \\ Complications Research Centre, Conway Institute, School of Medicine and Medical Science, University \\ College Dublin, Dublin, Ireland; ${ }^{5}$ Gastrosurgical Laboratory, Sahlgrenska Academy, University of Gothenburg, \\ Gothenburg, Sweden and ${ }^{6}$ Novo Nordisk Foundation Center for Basic Metabolic Research, Section for \\ Metabolic Receptology and Enteroendocrinology, Faculty of Health Sciences, University of Copenhagen, \\ Copenhagen, Denmark
}

\begin{abstract}
Roux-en-Y gastric bypass (RYGB) and duodenal jejunal bypass (DJB), two different forms of bariatric surgery, are associated with improved glucose tolerance, but it is not clear whether the gut microbiota contributes to this effect. Here we used fa/fa rats as a model of impaired glucose tolerance to investigate whether (i) the microbiota varies between fa/fa and nondiabetic fa/+ rats; (ii) the microbiota of fa/fa rats is affected by RYGB and/or DJB; and (iii) surgically induced microbiota alterations contribute to glucose metabolism. We observed a profound expansion of Firmicutes (specifically, Lactobacillus animalis and Lactobacillus reuteri) in the small intestine of diabetic fa/fa compared with nondiabetic fa/+ rats. RYGB-, but not DJB-, treated fa/fa rats exhibited greater microbiota diversity in the ileum and lower $L$. animalis and $L$. reuteri abundance compared with shamoperated fa/fa rats in all intestinal segments, and their microbiota composition resembled that of unoperated fa/+ rats. To investigate the functional role of RYGB-associated microbiota alterations, we transferred microbiota from sham- and RYGB-treated fa/fa rats to germ-free mice. The metabolic phenotype of RYGB-treated rats was not transferred by the transplant of ileal microbiota. In contrast, postprandial peak glucose levels were lower in mice that received cecal microbiota from RYGBversus sham-operated rats. Thus, diabetes-associated microbiota alterations in fa/fa rats can be modified by RYGB, and modifications in the cecal microbiota may partially contribute to improved glucose tolerance after RYGB.
\end{abstract}

The ISME Journal (2017) 11, 2035-2046; doi:10.1038/ismej.2017.70; published online 19 May 2017

\section{Introduction}

Roux-en-Y gastric bypass (RYGB) is an effective treatment for morbid obesity as well as type 2 diabetes (Carlsson et al., 2012). Diabetes remission is achieved within days of RYGB and before significant weight loss has been achieved (Brethauer et al., 2013; Sjostrom et al., 2014). Factors that have been proposed to contribute to the

Correspondence: F Bäckhed, Wallenberg Laboratory, Sahlgrenska Center for Cardiovascular and Metabolic Research, Department of Molecular and Clinical Medicine, Institute of Medicine, University of Gothenburg, Bruna Stråket 16, SE-413 45 Göteborg, Sweden. Email: Fredrik.Backhed@wlab.gu.se

${ }^{7}$ Current address: Novo Nordisk Foundation Center for Basic Metabolic Research, Section for Metabolic Receptology and Enteroendocrinology, Faculty of Health Sciences, University of Copenhagen, Copenhagen, Denmark.

Received 6 October 2016; revised 3 April 2017; accepted 7 April 2017; published online 19 May 2017 metabolic improvements following RYGB include calorie restriction (Isbell et al., 2010), increased postprandial release of glucagon-like peptide 1 (le Roux et al., 2006), enhanced circulating levels of bile acids (Simonen et al., 2012) and alterations in the metabolome (Mutch et al., 2009; Graessler et al., 2013a; Arora et al., 2015). In addition, RYGB has been shown to induce marked shifts in the intestinal microbiota in rodents (Li et al., 2011; Liou et al., 2013) and humans (Zhang et al., 2009; Kong et al., 2013; Graessler et al., 2013b; Tremaroli et al., 2015). However, it is not clear whether these alterations in the microbiota contribute to the improvement of glucose metabolism associated with RYGB.

Duodenal jejunal bypass (DJB) is a stomachsparing surgical procedure resulting in an intestinal configuration similar to that following RYGB (Figure 1a). DJB has been demonstrated to improve glucose tolerance in nonobese diabetic Goto-Kakizaki rats 
a

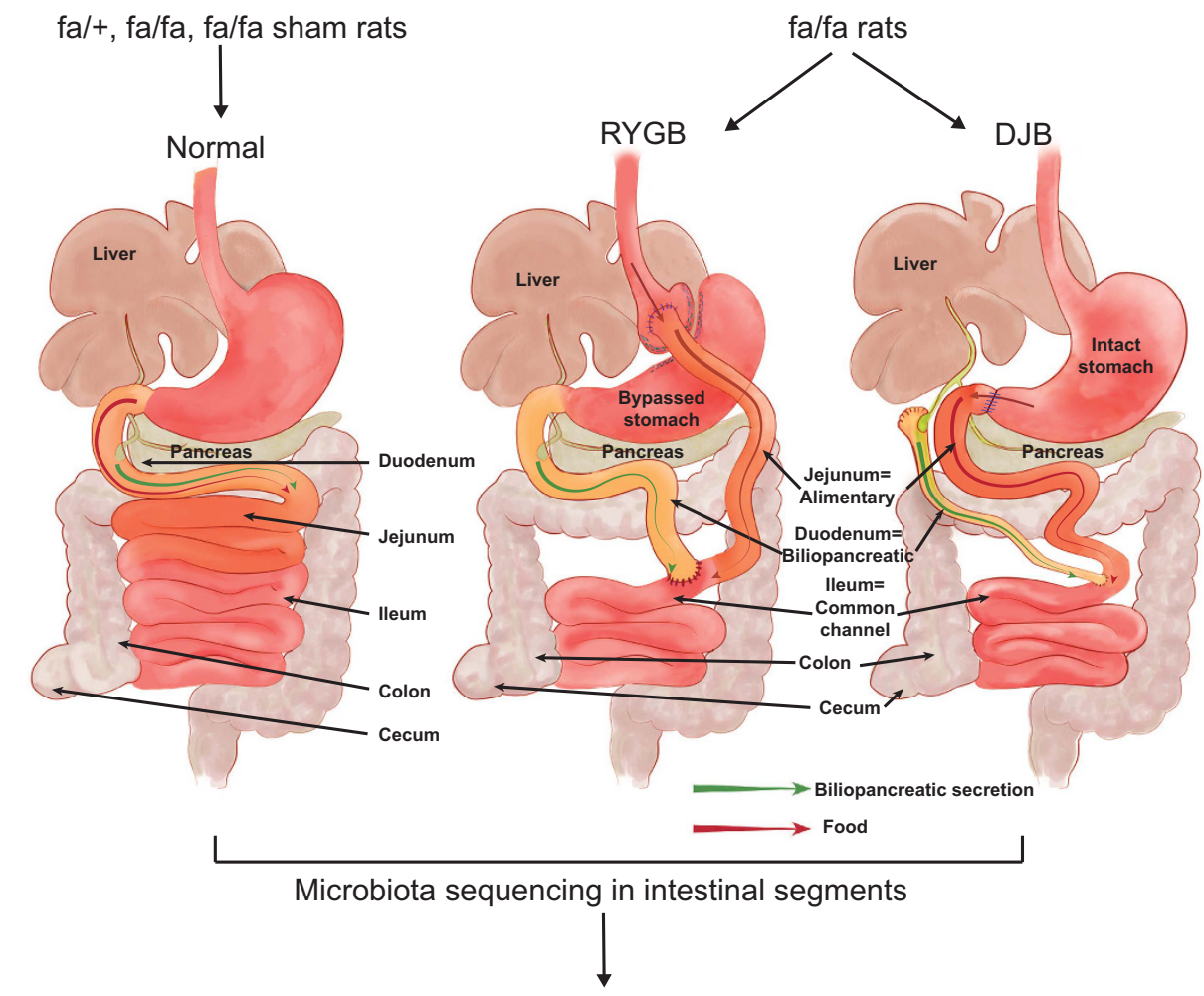

b Transplantation of ileal and cecal contents to germ-free mice

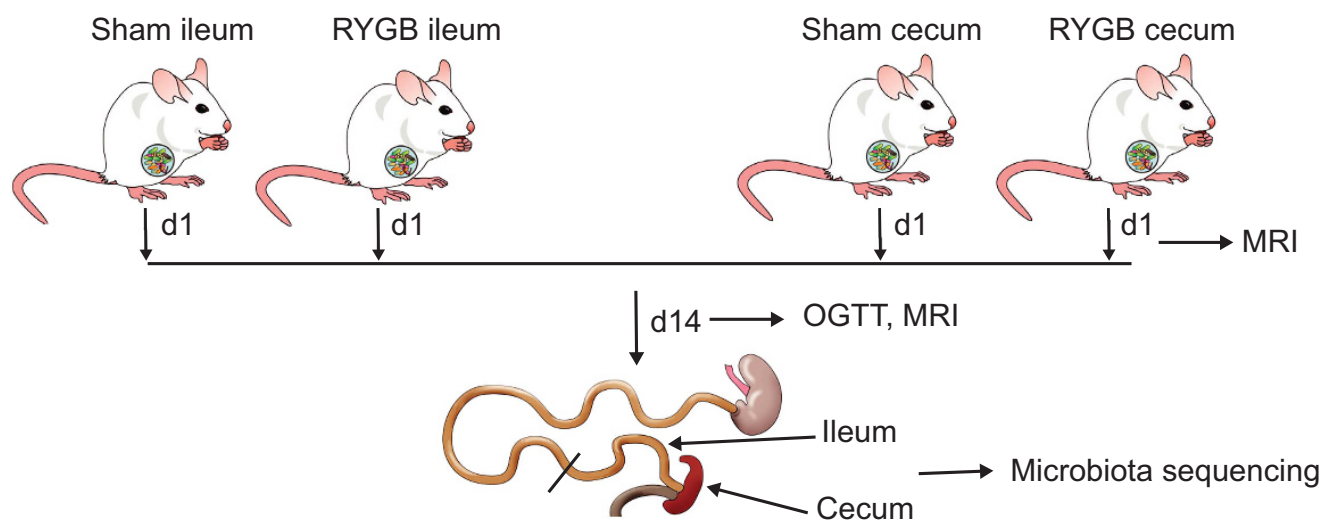

Figure 1 Schematic of experimental design. (a) Five groups of rats were used: unoperated fa/+ and fa/fa rats, and sham-operated, RYGBand DJB-treated fa/fa rats. In the sham group, gastrotomy on anterior wall of stomach and jejunostomy was performed with no reconfiguration of the gut. Intestinal segments were collected for microbiota sequencing. (b) Ileum and cecum from sham and RYGB rats (two donors each) were transferred to germ-free recipients ( $n=4-5$ per donor). Magnetic resonance imaging (MRI) was performed 1 and 14 days after transplantation. An oral glucose tolerance test (OGTT) was performed and ileum and cecum from each recipient were collected for microbiota sequencing 14 days after transplantation.

(Rubino et al., 2006; Kindel et al., 2009; Speck et al., 2011), although one study did not observe any improvement (Gavin et al., 2011). DJB has also been reported to promote mild improvements in glucose tolerance in overweight humans (Cohen et al., 2012; Klein et al., 2012). However, it is not known how this procedure affects the gut microbiota.

$\mathrm{Fa} / \mathrm{fa}$ rats are leptin receptor-deficient rats that develop impaired glucose tolerance with age (Ionescu et al., 1985). Earlier studies in fa/fa rats have reported that RYGB promotes pronounced weight loss and reduces glucose and insulin levels
(Xu et al., 2002) and DJB improves glucose tolerance (Rubino et al., 2005; Li et al., 2013; Patel et al., 2013). Thus, fa/fa rats represent an attractive animal model to study surgery-induced physiological improvements (Greenwood et al., 1982; Wolff et al., 2009; Lifante et al., 2012). Little is known about how the gut microbiota is linked to these changes in fa/fa rats, but a recent study reported that age and microenvironment affect the fecal microbiome in these rats (Lees et al., 2014). Here, we studied the gut microbiota of $\mathrm{fa} / \mathrm{fa}$ rats along the length of the intestine and investigated the following: (i) how the 
microbiota varies between diabetic fa/fa rats and nondiabetic fa/+ rats along the length of the intestine; (ii) whether changes in the gut microbiota in fa/ fa rats can be normalized by RYGB and/or DJB; and (iii) how the altered microbiota from different locations in the intestine contributes to glucose metabolism.

\section{Materials and methods}

Surgically treated rats

The five groups of rats used in the surgery experiments (fa/+, fa/fa, sham-operated fa/fa, RYGB-treated fa/fa and DJB-treated fa/fa rats) have been described in an earlier study to assess differences in glycemic control (Seyfried et al., 2014). Briefly, male fa/+ and fa/fa rats were purchased from Charles River, Lyon, France, at 6 weeks of age and were individually housed under ambient humidity at $22^{\circ} \mathrm{C}$ in a $12 \mathrm{~h}$ light/dark cycle. The rats had free access to drinking water and Purina Lab diet (Lab diet, St Louis, MO, USA, $16.7 \%$ of calories from fat). At 12 weeks of age, fa/fa rats were randomly allocated to an unoperated control group or to sham, RYGB or DJB surgery groups, and surgery was performed as described earlier (Seyfried et al., 2014). All experiments were approved by the Veterinary Office of the Canton Zurich, Switzerland.

Duodenal, jejunal and ileal contents from fa/+, fa/ fa and sham-operated groups and contents from corresponding segments (that is, biliopancreatic, alimentary and common channel limbs) of RYGBand DJB-treated fa/fa rats (Figure 1a) were collected on day 35 after surgery and snap frozen in liquid nitrogen immediately. Colon and cecal contents from all the groups were excised and snap frozen.

\section{Ileal and cecal transplants}

Germ-free Swiss Webster male mice aged 10 weeks were used for all transplantation experiments (Figure 1b). Frozen contents from the ileum and cecum of sham- and RYGB-operated fa/fa rats were homogenized in phosphate-buffered saline buffer supplemented with reducing solution $\left(0.02 \mathrm{M} \mathrm{Na}_{2} \mathrm{~S}\right.$ and $1 \%$ cystein dissolved in $\mathrm{NaHCO}_{3}$ buffer). Two separate donors from each group were selected. Germ-free mice (four to five mice per group) were fasted for $4 \mathrm{~h}$ and gavaged with the resultant slurry $(200 \mu l)$. The transplanted mice were maintained in autoclaved individual ventilated cages with sterile bedding and fed autoclaved food and water ad libitum for 2 weeks. Whole-body magnetic resonance imaging was performed on day 1 and day 14 after the transplantation. On day 14, an oral glucose tolerance test (OGTT) was performed in mice by oral administration of $30 \%$ D-glucose $\left(2 \mathrm{~g} \mathrm{~kg}^{-1}\right.$ body weight) after a $4 \mathrm{~h}$ fast. Blood was drawn from the tail vein at $0,15,30,60,90$ and $120 \mathrm{~min}$, and blood glucose levels were measured with a Bayer glucometer.
The ileum and cecum from colonized mice were collected and snap frozen in liquid nitrogen and stored at $-80^{\circ} \mathrm{C}$ until further process. These procedures were approved by the Ethics Committee on Animal Care and Use in Gothenburg, Sweden.

\section{Gut microbiota analysis}

DNA was extracted from all the rat samples using repeated bead-beating protocol as described previously (Salonen et al., 2010), and the V1-V2 region of bacterial 16S rRNA gene was amplified using the $27 \mathrm{~F}$ and barcoded $338 \mathrm{R}$ primers fused with 454 Titanium sequencing adaptors (Hamady et al., 2008). Three independent $25 \mu \mathrm{l}$ PCR reactions were performed for each sample under the following conditions: one cycle: $3 \mathrm{~min}$ at $95^{\circ} \mathrm{C}$; 30 cycles: $20 \mathrm{~s}$ at $95^{\circ} \mathrm{C}$, $30 \mathrm{~s}$ at $52{ }^{\circ} \mathrm{C}$, and $60 \mathrm{~s}$ at $72{ }^{\circ} \mathrm{C}$ and $10 \mathrm{~min}$ at $72^{\circ} \mathrm{C}$. The resulting product was checked for size and purity on $1 \%$ agarose gel, further purified (NucleoSpin 740609, Macherey-Nagel, Düren, Germany), and quantified with the Quant-iT PicoGreen dsDNA kit (Invitrogen, Carlsbad, CA, USA). Low-yield samples were speed vacuumed to concentrate DNA. All the samples were pooled in equal amounts $\left(20 \mathrm{ng}^{-1}\right)$ and purified again with magnetic beads (AMPure XP, Beckman, Danvers, MA, USA) to remove short amplification products. The purified amplicons were sequenced in a 454 GS FLX system with Titanium chemistry by GATC Biotech (Konstanz, Germany). Two samples with low sequences were excluded from analysis.

DNA was extracted from the mouse samples by the same protocol as above. The V4 region of the $16 \mathrm{~S}$ rRNA gene was amplified using $515 \mathrm{~F}$ and $806 \mathrm{R}$ primers designed for dual indexing (Kozich et al., 2013), $10 \mathrm{ng}^{-1}$ samples were pooled (with the same purification steps) and the Illumina MiSeq (Illumina RTA v1.17.28; MCS v2.5) platform was used for the analysis.

Quality-filtered 454 pyrosequencing and Illumina reads from both experiments were analyzed using the QIIME (Quantitative Insights Into Microbial Ecology) software package (Caporaso et al., 2010a; version 1.9.1) as described previously (Caesar et al., 2015). Briefly, chimeric sequences were identified with ChimeraSlayer (Haas et al., 2011) and excluded from all downstream analyses. The sequences were clustered into operational taxonomic units (OTUs) at a 97\% identity threshold using a closed-reference OTU picking approach with UCLUST (Edgar, 2010) against the Greengenes reference database (DeSantis et al., 2006). Representative OTUs were aligned using PyNAST (Caporaso et al., 2010b) and used to build a phylogenetic tree which was used to estimate $\alpha$-, and $\beta$-diversity of samples using unweighted UniFrac (Lozupone and Knight, 2005). Sequences that could not be aligned with PyNAST and singletons were excluded. To correct for differences in sequencing depth, the same number of sequences were randomly sub-sampled for each sample and 
used for diversity analyses. Statistical significance of sample groupings was tested with a multivariate nonparametric analysis of variance (Adonis, 999 permutations; Anderson, 2001). Sequence data from the rat and mice analyses are stored in the European Nucleotide Archive (www.ebi.ac.uk/ena) under accession numbers PRJEB20172 and PRJEB20197, respectively.

The online source BLAST was used to identify V1-V2 sequences at the species level for the most abundant and significant OTUs that had not already been specified in the OTU table.

\section{Statistical analysis}

Data are presented as mean \pm s.e.m. OTUs (at six taxonomic levels, from phylum to species level) differing between groups were identified in QIIME with the nonparametric Kruskal-Wallis test. To determine the differences between groups (which were not reported in QIIME), the OTUs that had a $P$-value $<0.05$ (with false discovery rate correction) in the QIIME analysis were thereafter analyzed with Kruskal-Wallis test with Dunn's multiple comparison to determine pair-wise differences using GraphPad prism version 6 (GraphPad Software Inc.) as reported previously (Fak et al., 2015). Statistical comparison of two groups was performed by the nonparametric Mann-Whitney $U$-test. Significance was established at $P<0.05$.

\section{Results}

Composition of the gut microbiota of fa/fa rats is modified by $R Y G B$ but not by DJB

Fasting glucose and insulin levels in the five groups of rats used in this study (unoperated fa/+, unoperated fa/fa, sham-operated fa/fa, RYGB-treated fa/fa and DJB-treated fa/fa) have previously been reported: both variables were significantly higher in unoperated fa/fa rats compared with fa/+ rats at 17 weeks of age and were normalized in fa/fa rats that underwent RYGB but not in those that were sham operated (Seyfried et al., 2014). There was a small reduction in fasting blood glucose levels in DJB-treated fa/fa rats compared with sham-operated rats, but levels remained significantly higher than those in RYGB-treated rats (Seyfried et al., 2014). Body weight was similar in all groups of mice at 17 weeks of age except for the RYGB-treated group in which body weight was significantly lower (Seyfried et al., 2014).

To determine whether these differences between the groups of rats were associated with changes in the microbiota along the length of the gut, we performed 454 sequencing on samples collected from the duodenum, jejunum, ileum and colon of these rats. In total, we generated 566521 reads averaging $5665 \pm 2086$ reads per sample. Alpha diversity (as measured by the observed species) did not differ between fa/fa and fa/+ rats nor between DJB-treated rats and sham-operated rats but was significantly higher in the ileum of RYGB-treated rats compared with sham-operated rats (Figure 2a). Unweighted UniFrac analysis (sensitive to the phylogenetic relatedness of taxa) on all the samples revealed significant clustering by intestinal segment (Adonis: $R^{2}=0.12, P=0.001$; Figure 2b). This analysis also showed that surgery could explain more variability in the microbiota composition (Adonis: $\left.R^{2}=0.42, \quad P=0.001\right)$ than intestinal segment; in particular, RYGB samples formed a separate cluster (Figure 2c). The non-phylogenetic Bray-Curtis analysis showed similar results (Supplementary Figure S1). We also performed unweighted UniFrac analysis on the samples divided according to intestinal segment and observed significant clustering of samples from RYGB-treated fa/fa rats in the duodenum (Adonis: $R^{2}=0.28, P=0.001$ ), jejunum (Adonis: $\quad R^{2}=0.34, \quad P=0.001$ ), ileum (Adonis: $R^{2}=0.41, P=0.001$ ) and colon (Adonis: $R^{2}=0.31$, $P=0.001$; Figure 2d).

Firmicutes was the most abundant phylum in the small intestinal regions of unoperated fa/+ rats, and increased in abundance in unoperated fa/fa rats to $98 \%$ of the total microbial composition in the jejunum and ileum (Figure 3a). The levels of Firmicutes were not affected by DJB compared with sham surgery in the small intestine of fa/fa rats, but were significantly lower in fa/fa rats after RYGB versus sham in the ileum (Figure 3a). Bacteroidetes was the second-most abundant phylum in the small intestinal regions of unoperated fa/ + rats and it showed a trend towards lower abundance in the duodenum, jejunum and ileum of unoperated $\mathrm{fa} / \mathrm{fa}$ compared with fa/+ rats (Figure 3a). The levels of Bacteroidetes were not affected by DJB compared with sham surgery in the jejunum and ileum of fa/fa rats, but were significantly higher in fa/fa rats after RYGB versus sham in the ileum (Figure 3a). The abundance of the phylum Proteobacteria was significantly higher in the jejunum of unoperated fa/+ rats and RYGB-treated fa/fa rats compared with sham-operated fa/fa rats (Figure $3 \mathrm{a}$ ).

By comparing the relative abundance of genera across the five groups of rats and along the length of the intestine, we showed that Lactobacillus was the predominant genus in all the intestinal regions in unoperated fa/fa, and sham-operated and DJB-treated rats (Supplementary Figure S2). By contrast, Lactococcus was the most abundant genus in RYGB-treated rats in all the intestinal regions (Supplementary Figure S2).

Supplementary Table S1 shows the taxa that significantly differed in abundance in unoperated $\mathrm{fa} /+$ rats and RYGB-treated fa/fa rats compared with sham-operated fa/fa rats. Using this information, we focused on the most abundant bacterial species from the three most predominant phyla to identify key species that could potentially contribute to the observed differences in phenotype between the groups of rats. The taxa in Supplementary Table S1 included OTUs that had not earlier been identified at 


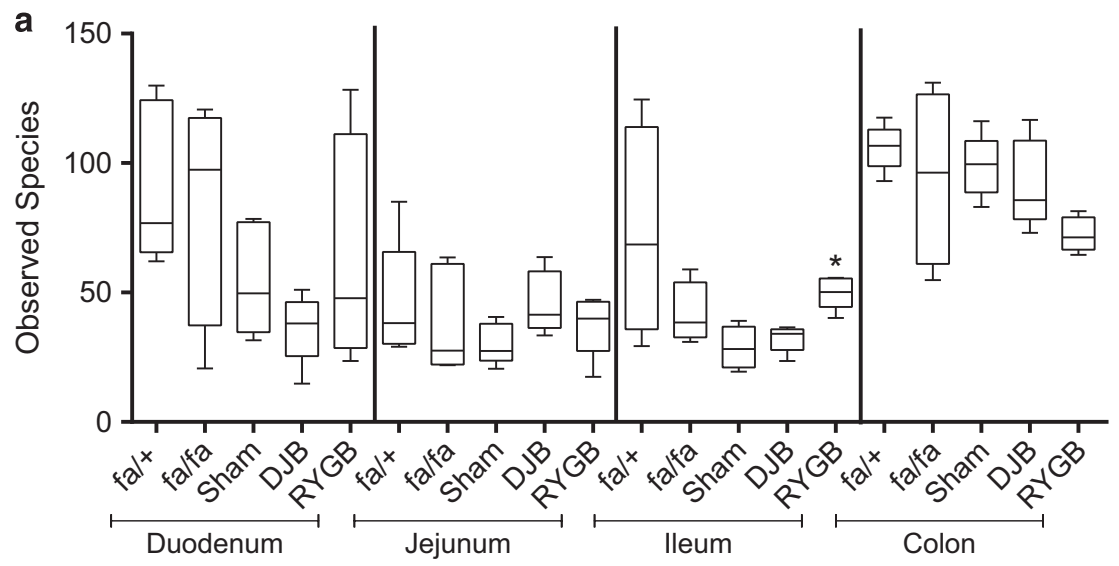

b

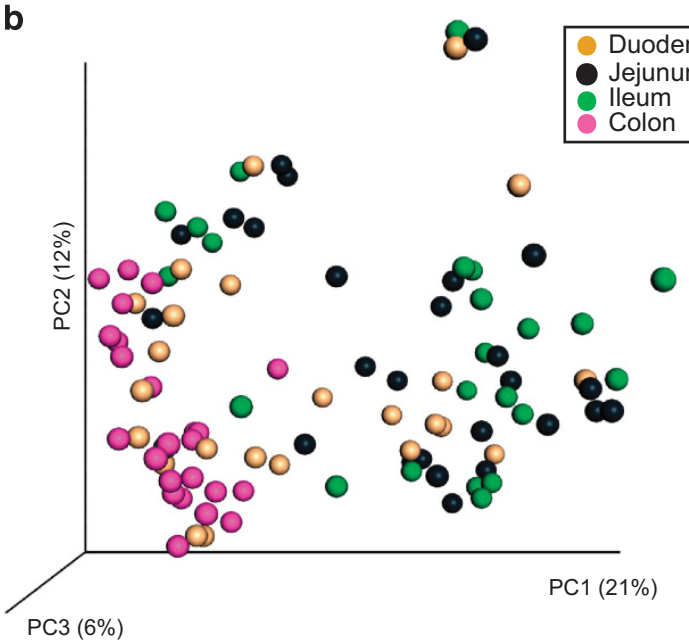

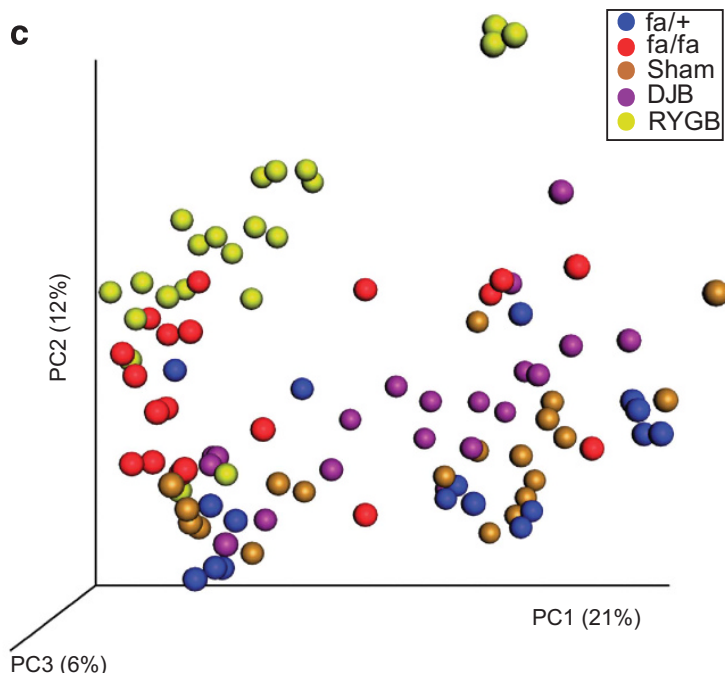

d

Duodenum

Jejunum

Ileum

Colon
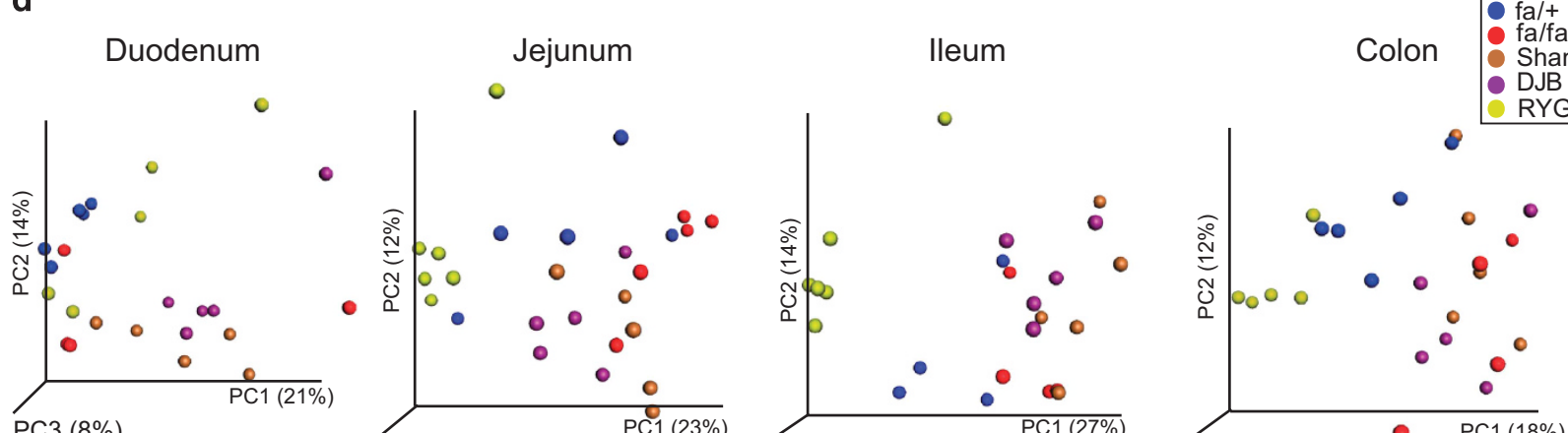

○
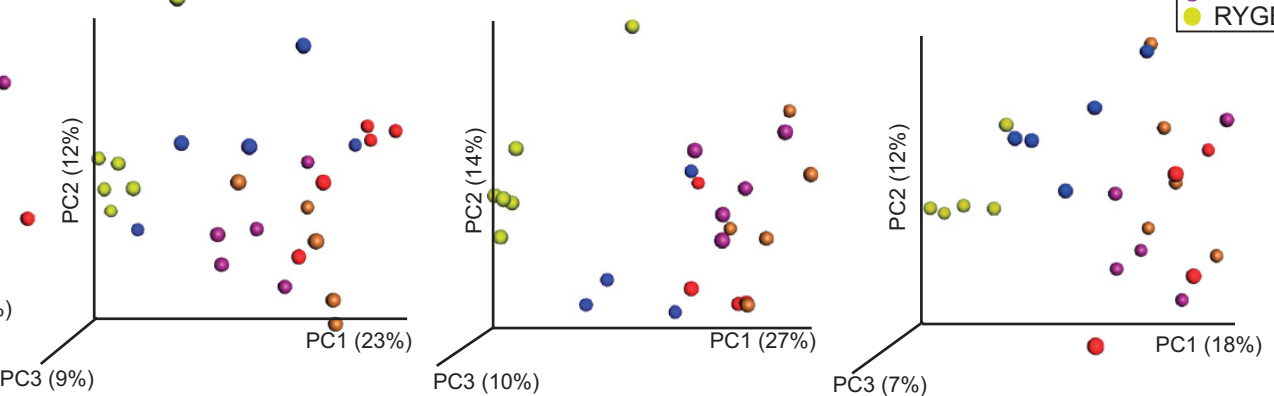

Figure 2 Bacterial diversity along the length of intestine in unoperated and operated fa/fa rats. (a) Diversity box plots obtained from alpha rarefaction measured by observed species. Data are shown as box and whiskers plots, indicating the median (thick line in the box), the 25th-75th percentile range (boxes), and the 1st-99th percentile range (whiskers). ${ }^{*} P<0.05$ versus sham in ileum. Principal coordinate analysis (PCoA) plot using unweighted UniFrac distances of gut microbial communities separated either by (b) intestinal segment; (c) surgery; or (d) as in c, but divided according to intestinal segment in fa/+ $(n=4-5)$, fa/fa $(n=4-5)$, sham-operated fa/fa $(n=5)$, DJBtreated fa/fa $(n=5)$ and RYGB-treated fa/fa $(n=5-6)$ rats. The first three prinicipal coordinates from PCoA are plotted. Symbols represent data from individual rats.

the species level, and we used BLAST to identify the most abundant OTUs within Firmicutes, Bacteroidetes and Proteobacteria at the species level. Thus, OTU 189681 was identified as Lactobacillus animalis $(\max$ score $=2169$, total score $=2169$, query cover $=100 \%$, identity $=94 \%, E$-value $=0.0)$; OTU
199716 was identified as Bacteroides vulgatus (max score $=2128$, total score $=14827$, query cover $=100 \%$, identity $=94 \%, E$-value $=0.0$ ); and OTU 1109362 was identified as Escherichia coli (max score $=2525$, total score $=17379$, query cover $=100 \%$, identity $=97 \%$, $E$-value $=0.0$ ). 
Within Firmicutes, the most abundant species that differed across the groups of rats were $L$. animalis, Lactobacillus reuteri and Lactococcus sp.
(Figures 3b-d). Both L. animalis and L. reuteri showed a similar pattern in all the intestinal segments investigated, with increased abundance in
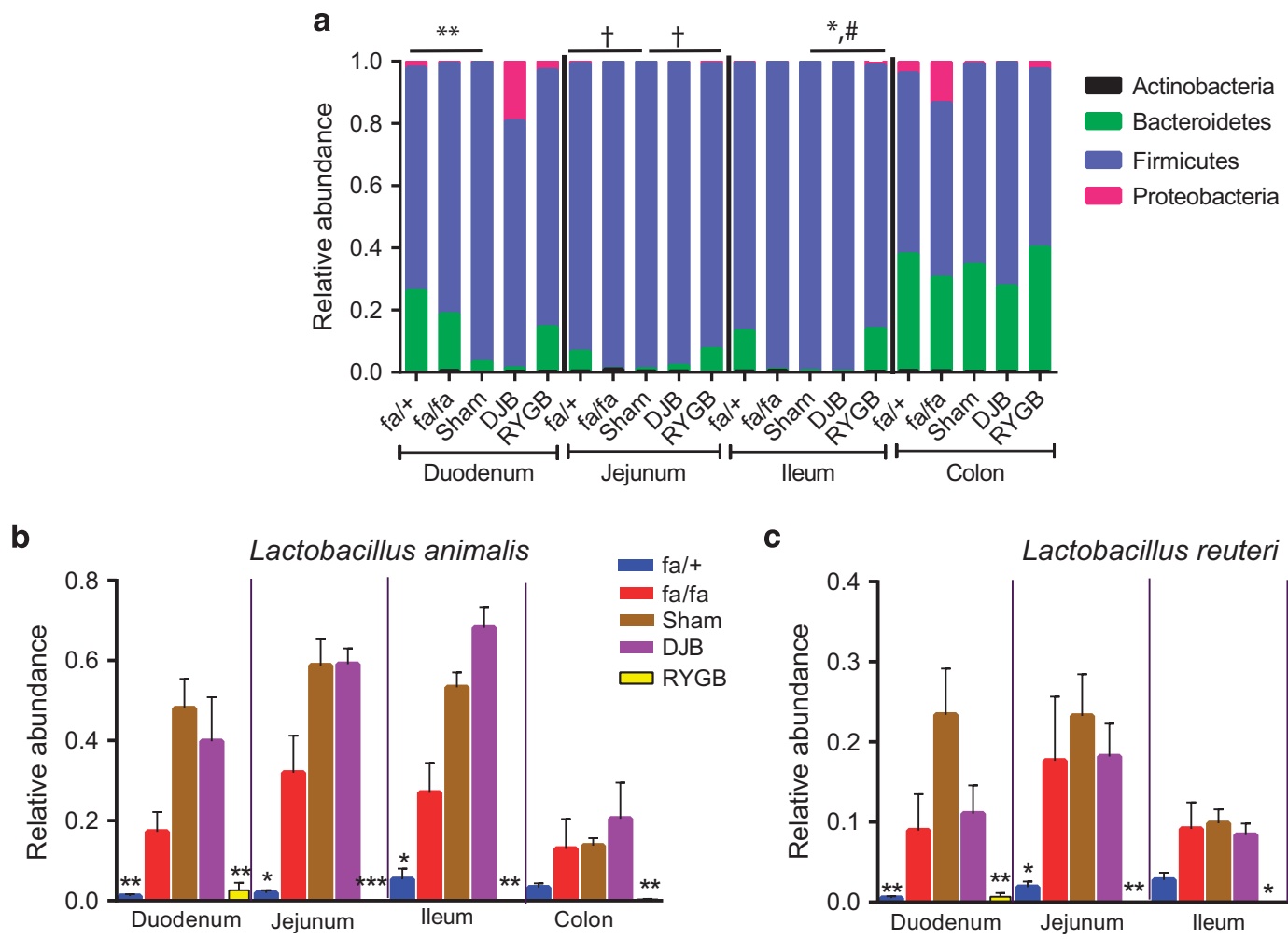

C
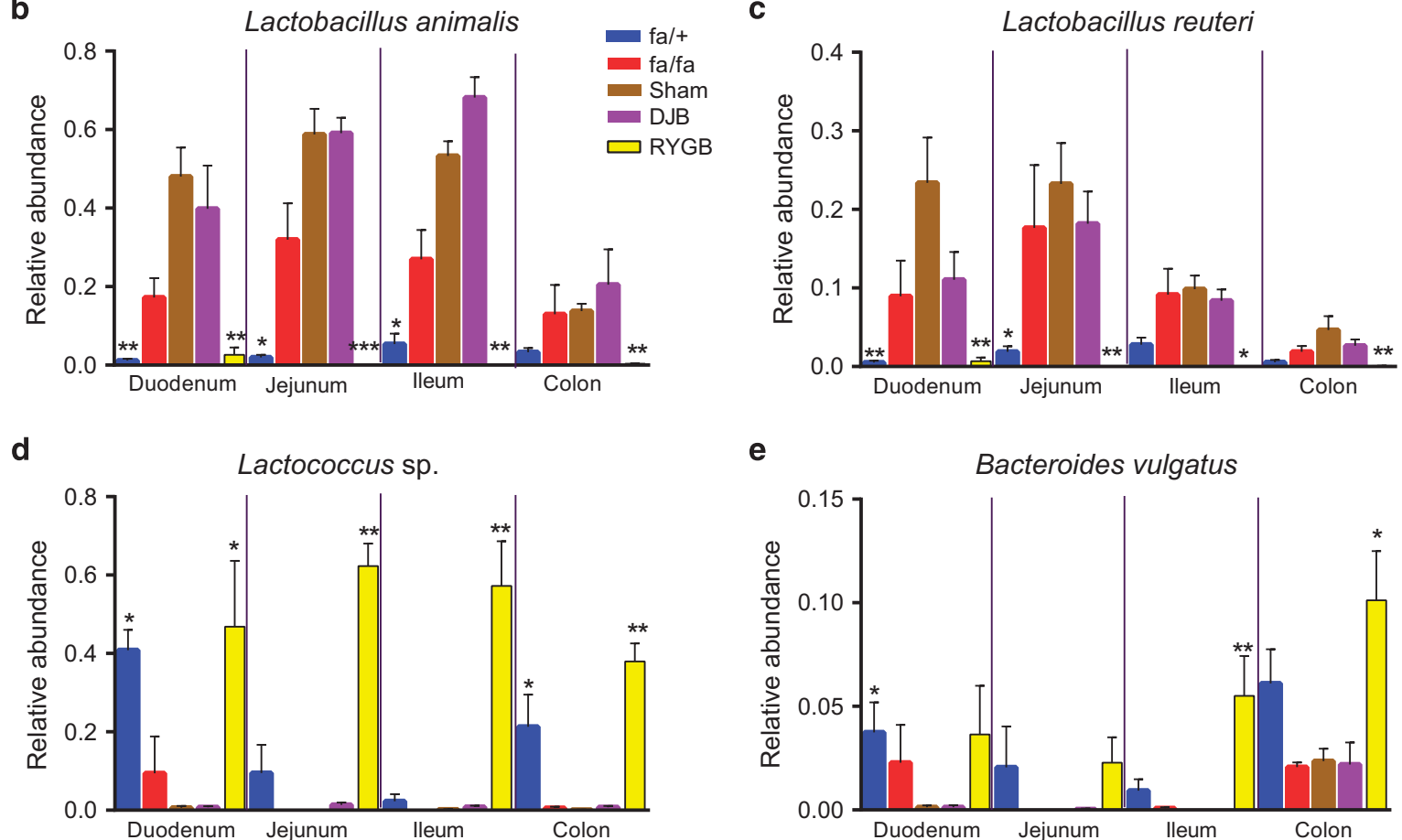

e

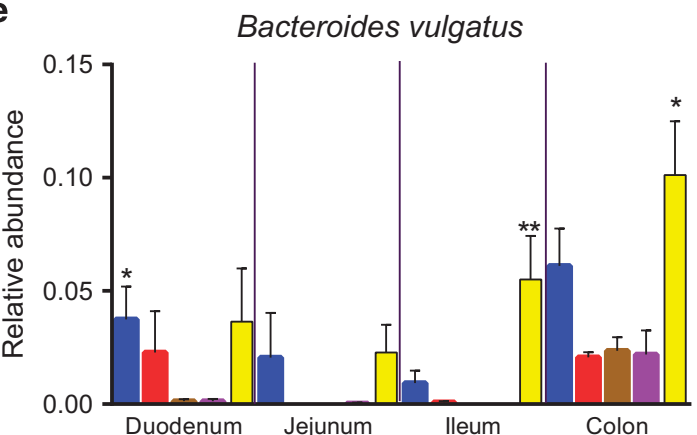

f

Bacteroides acidifaciens

g

Escherichia coli
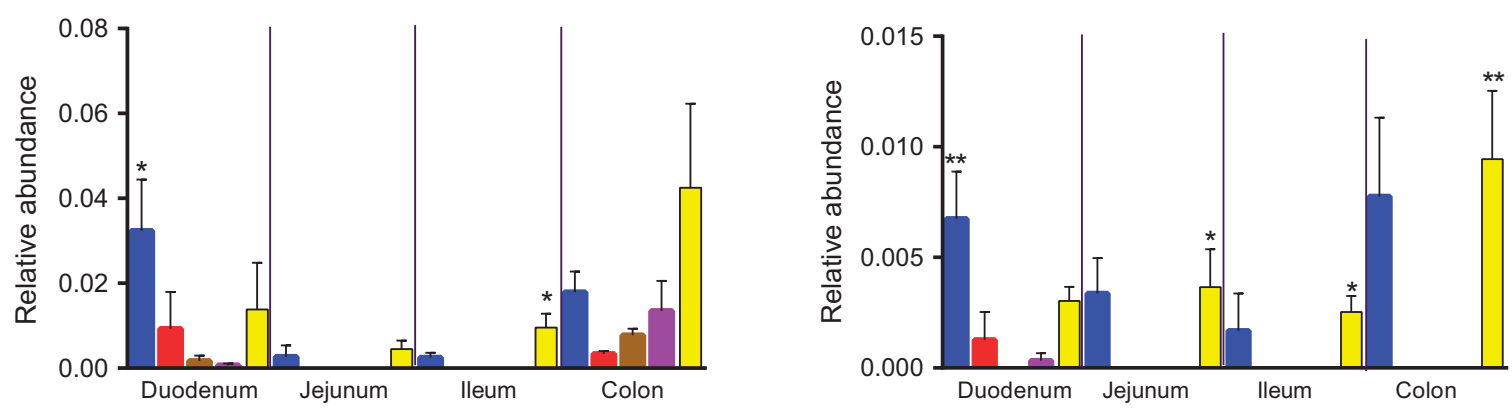

Figure 3 Microbial community shifts along the length of intestine in unoperated and operated fa/fa rats. Relative abundance of (a) major phyla, (b) Lactobacillus animalis, (c) Lactobacillus reuteri, (d) Lactococcus sp., (e) Bacteroides vulgatus, (f) Bacteroides acidifaciens and (g) Escherichia coli in duodenum, jejunum, ileum and colon of fa/+ $(n=4-5)$, fa/fa $(n=4-5)$, sham-operated fa/fa $(n=5)$, DJB-treated fa/fa $(n=5)$ and RYGB-treated fa/fa $(n=5-6)$ rats. Data are mean \pm s.e.m. In $\mathbf{a},{ }^{*} P<0.05,{ }^{* *} P<0.01$ for Bacteroidetes, ${ }^{*} P<0.05$ for Firmicutes and ${ }^{+} P<0.05$ for Proteobacteria. In $\mathbf{b}-\mathbf{g},{ }^{*} P<0.05$, ${ }^{* *} P<0.01$, ${ }^{* * *} P<0.001$ versus sham in respective intestinal segments based on nonparametric Kruskal-Wallis test with Dunn's multiple comparison analysis. 
sham-operated fa/fa rats compared with unoperated $\mathrm{fa} /+$ rats, no difference between DJB-treated and shamoperated fa/fa rats, and significant reductions in RYGB-treated compared with sham-operated fa/fa rats (Figures $3 \mathrm{~b}$ and c). Lactococcus sp. also showed a similar pattern in all the intestinal segments investigated, but its abundance was dramatically reduced in sham-operated fa/fa rats compared with unoperated fa/+ rats, not affected by DJB compared with sham surgery, and dramatically increased in RYGB-treated compared with sham-operated fa/fa rats (Figure 3d).

Within Bacteroidetes, the most abundant species that differed across the groups of rats were $B$. vulgatus and Bacteroides acdifaciens (Figures $3 \mathrm{e}$ and f). The abundance of these species was reduced in shamoperated fa/fa rats compared with unoperated fa/+ rats in the duodenum, not affected by DJB compared with sham surgery and increased in RYGB-treated compared with sham-operated fa/fa rats, and the differences were most pronounced in ileum (Figures 3e and f). Among Proteobacteria, E. coli also increased in the jejunum, ileum and colon in RYGB-treated compared with sham-operated fa/fa rats (Figure $3 \mathrm{~g}$ ).
Thus, RYGB surgery in fa/fa rats promoted changes in the microbial composition along the length of the intestine, in particular, a dramatic reduction in the abundance of Lactobacillus species and an increase in Bacteroides species, resulting in a microbiota composition similar to that of unoperated nondiabetic fa/+ rats. These changes (compared with sham surgery) were not observed following DJB in fa/fa rats.

Transplanting the ileal microbiota does not transfer the metabolic phenotype of fa/fa rats

As RYGB had the largest effect on the microbiota in the ileum, we transferred the ileal microbiota of sham- and RYGB-operated fa/fa rats to germ-free mice to investigate whether the ileal microbiota directly contributes to the metabolic phenotype of these rats. Alpha diversity was lower in the cecum of germ-free mice that received ileal microbiota from sham-operated rats (termed 'sham recipients') compared with germ-free mice that received ileal microbiota from RYGB-treated rats (termed 'RYGB recipients'; Figure 4a). Unweighted UniFrac analysis
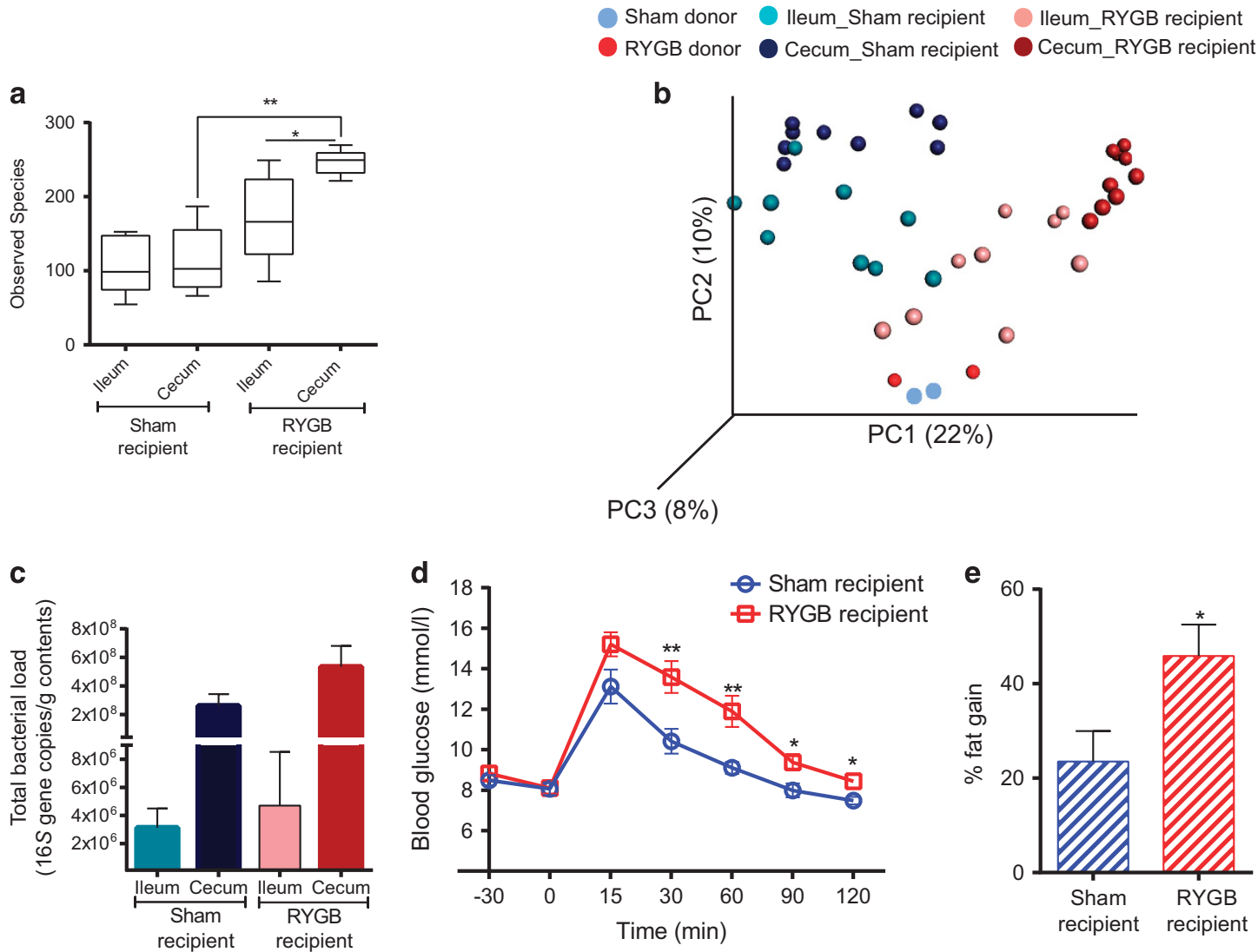

Figure 4 Ileal microbiota transplant. (a) Bacterial diversity as shown by diversity box plots obtained from alpha rarefaction measured by observed species. Data are shown as box and whiskers plots, indicating the median (thick line in the box), the 25th-75th percentile range (boxes) and the 1st-99th percentile range (whiskers). (b) Principal coordinate analysis (PCoA) plot using unweighted UniFrac distances of gut microbial communities obtained from sham and RYGB donors $(n=2)$ and ileum and cecum of mice $(n=9)$ colonized with ileal microbiota from sham-operated or RYGB-treated fa/fa rats. Symbols represent data from individual rat donors or mouse recipients. (c) DNA copies of total bacteria per gram contents in the ileum and cecum of mice $(n=9)$ colonized with ileal microbiota from sham-operated or RYGB-treated fa/fa rats. (d) Oral glucose tolerance test curves and (e) fat percentage gain in mice $(n=9)$ colonized with ileal microbiota from sham-operated or RYGB-treated fa/fa rats. Data are mean \pm s.e.m. ${ }^{*} P<0.05,{ }^{*} P<0.01,{ }^{* *} P<0.001$ as indicated or versus sham recipient. 
showed separation of sham and RYGB recipients along the first principal component (Adonis: $R^{2}=0.37, P=0.001$, Figure $4 \mathrm{~b}$ ), indicating that the donor ileal microbiota determines the microbiota composition of the recipient.

Despite the lower microbial diversity in the recipients of sham compared with RYGB ileal microbiota, levels of colonization (that is, total bacteria) were similar in both the groups (Figure 4c), with higher bacterial load in the cecum compared with the ileum. Glucose tolerance (in response to an OGTT) was impaired and fat gain was higher in the RYGB recipients compared with the sham recipients (Figures $4 \mathrm{~d}$ and e).

Transplanting the cecal microbiota transfers the metabolic phenotype of fa/fa rats

To investigate whether the microbiota in the cecum, which has a higher biomass and is more metabolically active compared with the ileal microbiota, of fa/fa rats directly contributes to the metabolic phenotype of these rats, we transplanted the cecal microbiota of sham- and RYGB-operated fa/fa rats into germ-free mice and colonized the mice for 2 weeks.

Alpha diversity was lower in the cecum of recipients of cecal microbiota from sham- versus RYGB-operated fa/fa rats (Figure 5a). Unweighted UniFrac analysis confirmed that samples clustered according to the type of donor microbiota (Adonis: $R^{2}=0.38, P=0.001$, Figure $5 \mathrm{~b}$ ).

There were no differences in the total levels of bacteria in the ileum or cecum of sham versus RYGB recipient mice (Figure 5c). However, the peak glucose concentration in the OGTT was higher in the sham recipients than in the RYGB recipients, indicating improved glucose tolerance in the RYGB recipients (Figure 5d). Adiposity in sham and RYGB recipients 2 weeks after transplantation was similar (Figure 5e).

\section{Discussion}

We previously investigated the effect of surgery on glycemic control in diabetic fa/fa rats and showed
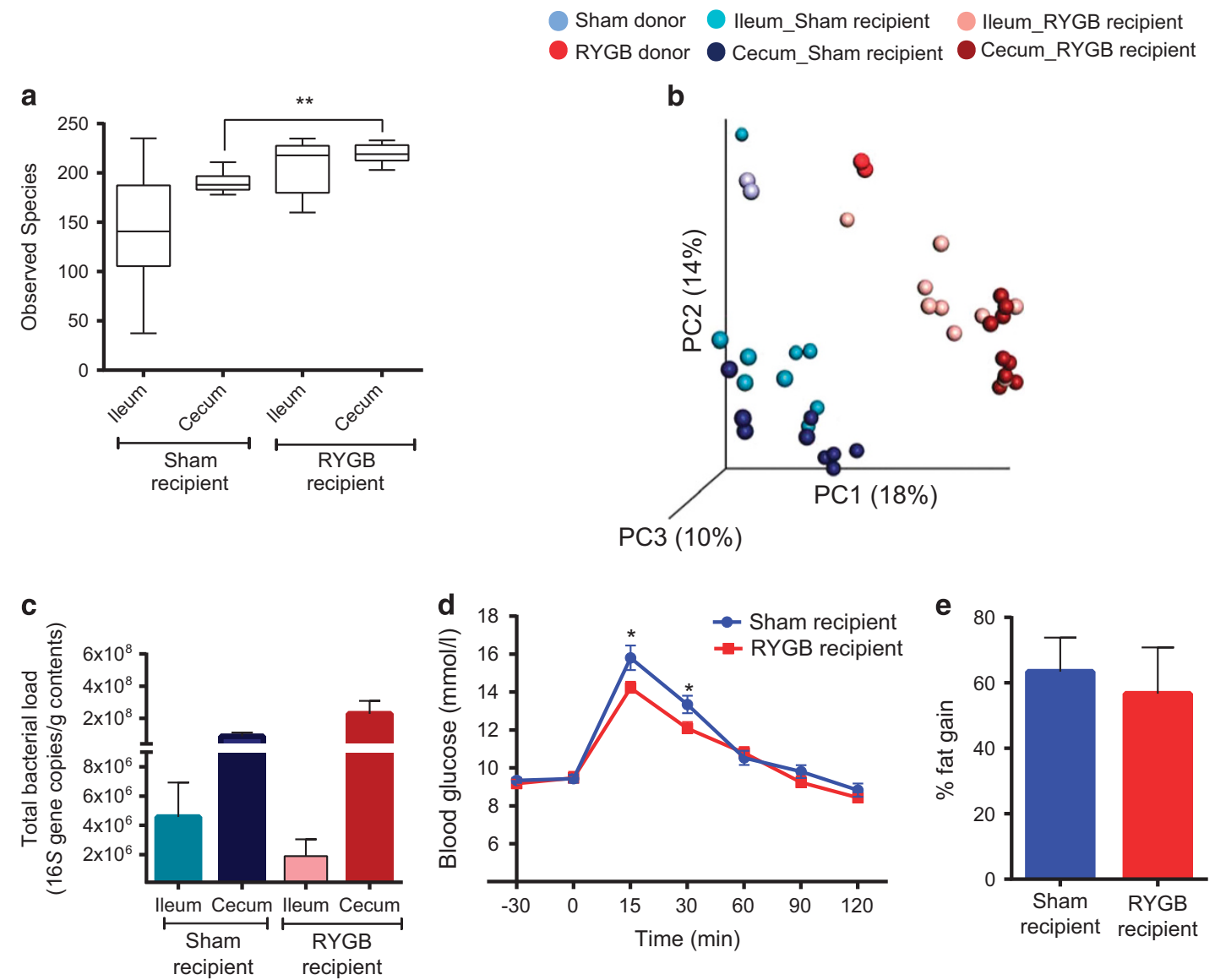

Figure 5 Cecal microbiota transplant. (a) Bacterial diversity as shown by diversity box plots obtained from alpha rarefaction measured by observed species. Data are shown as box and whiskers plots, indicating the median (thick line in the box), the 25th-75th percentile range (boxes), and the 1st-99th percentile range (whiskers). (b) Principal coordinate analysis (PCoA) plot using unweighted UniFrac distances of gut microbial communities obtained from sham and RYGB donors $(n=2)$ and ileum and cecum of mice $(n=9)$ colonized with cecal microbiota from sham-operated and RYGB-treated rats. Symbols represent data from individual rat donors and mouse recipients. (c) DNA copies of total bacteria per gram contents in the ileal and cecal microbiota of mice $(n=9)$ colonized with cecal microbiota from shamoperated and RYGB-treated rats. (d) Oral glucose tolerance test curves and (e) fat percentage gain in mice $(n=9)$ colonized with cecal microbiota from sham or RYGB rats. Data are mean \pm s.e.m. ${ }^{*} P<0.05$, ${ }^{*} P<0.01$ as indicated or versus RYGB recipient. 
that (i) RYGB produces significant weight loss and improved fasting glucose levels compared with sham surgery; and (ii) DJB produces mild improvements in fasting blood glucose without weight loss (Seyfried et al., 2014). Here we studied the microbiota composition of these rats along the length of the intestine to investigate potential associations between the microbiota and glycemic control. First, we showed an expansion of Firmicutes and reduction in Bacteroidetes in the jejunum and ileum of $\mathrm{fa} / \mathrm{fa}$ rats compared with nondiabetic fa/+ rats. Second, we observed that RYGB, but not DJB surgery, in fa/fa rats promoted changes in the microbial composition along the length of the intestine; in particular, a dramatic reduction in the abundance of Lactobacillus species (including $L$. reuteri and L. animalis) and an increase in Lactococcus and Bacteroides species, resulting in a microbiota composition similar to that of unoperated nondiabetic fa/ + rats. Finally, by microbiota transfer to germ-free mice, colonization with the cecal, but not the ileal, microbiota of RYGB-treated fa/fa rats partially transferred the phenotype of improved glycemic control to recipient mice.

An association between Lactobacillus species and blood glucose levels has previously been noted in humans. For example, an increased abundance of Lactobacillus species has been reported in both European (Karlsson et al., 2013; Marlene et al., 2013) and Chinese (Le et al., 2012; Qin et al., 2012) patients with type 2 diabetes, and fecal Lactobacillus levels were shown to correlate with blood glucose levels in a study of men with varying degrees of glucose tolerance (Larsen et al., 2010). However, it is not known whether Lactobacillus contributes to type 2 diabetes or increases merely as a consequence of the disease. Indeed, Lactobacillus species are used as probiotics and have been shown to improve glucose levels in mice (Yakovlieva et al., 2015).

Although the intestinal configuration following RYGB and DJB is similar (Figure 1), DJB did not affect the microbiota composition compared with sham. Microbiota changes in response to RYGB are likely to be produced by reduced calorie intake, changes in luminal $\mathrm{pH}$ and an early arrival of undigested food in the alimentary limb. RYGB is also accompanied by increased expression of glucose transporter 1 in rats (Saeidi et al., 2013) and humans (Nguyen et al., 2014), which facilitates increased glucose uptake in the alimentary limb thus reducing simple sugars in the intestinal lumen, which may explain the reduced levels of Lactobacillus. By contrast, DJB is a stomach-sparing procedure, and thus gastric juices, stomach size in addition to calorie intake are likely important factors in shaping the gut microbiota.

The important contribution of a microbiota to metabolic phenotype was first shown in germ-free C57/BL6 mice that gained $57 \%$ of fat and exhibited impaired glucose tolerance after 2 weeks of colonization with cecal microbiota from a conventionally raised mouse (Backhed et al., 2004), likely due to the extra energy provided by utilization of indigestible carbohydrates by a complex gut microbiota. More recent studies have shown that metabolic phenotypes can be transferred by transplanting the microbiota. For example, a reduction in adiposity after RYGB can be transferred by transplanting cecal contents from RYGB-treated mice (Liou et al., 2013) or feces from RYGB-operated humans (Tremaroli et al., 2015) into germ-free mice; however, these studies did not report an effect of microbiota transfer on glucose tolerance. Although a causal relationship between altered gut microbiota and improved glucose control after RYGB is uncertain in humans, it has been reported that fecal transplant from lean donors to men with metabolic syndrome improved insulin sensitivity and increased populations of butyrate-producing intestinal bacteria (Vrieze et al., 2012).

In our study, the differences between the microbiota of sham- versus RYGB-treated fa/fa rats were higher in the ileum than in the colon, and we therefore speculated that the difference in metabolic phenotype between these groups could be transferred by transplanting the ileal microbiota. However, we showed that colonization of germ-free mice with ileal microbiota from shamoperated rats resulted in less fat gain and improved glucose tolerance compared with recipients of ileal microbiota from RYGB-treated rats. This could potentially be explained by the lower diversity in sham versus RYGB donor ileal microbiota, a phenotype that was transferred to recipients. Microbiota with low bacterial diversity may be less adapted to metabolize complex carbohydrates resulting in lower fat gain and improved glucose tolerance.

In contrast to mice colonized with ileal microbiota, mice colonized with cecal microbiota from shamoperated rats showed worse glucose tolerance compared with recipients of cecal microbiota from RYGB-treated rats. Although microbial diversity was lower in the cecum of recipients of cecal microbiota from sham- versus RYGB-operated rats, it was not as low as in the recipients of ileal microbiota from sham-operated rats. Thus, bacterial diversity may not be a limiting factor in the cecal transfer experiments. These results suggest that differences in cecal microbiota composition between the sham- versus RYGB-operated rats could contribute to the improved metabolic phenotype of RYGB-operated rats.

Taken together, our results showed that the microbiota composition of diabetic fa/fa rats along the length of intestine was significantly altered by RYGB but not by DJB, and resembled the microbiota composition of fa/+ rats. Importantly, we also showed that RYGB-induced effects on the cecal microbiota partly contributed to improved glucose tolerance. 


\section{Conflict of Interest}

FB is the founder and shareholder of Metabogen AB. The remaining authors declare no conflict of interest.

\section{Acknowledgements}

We thank Anna Hallen for producing Figure 1 and Petia Kovatcheva for critical comments on the manuscript. The computations for pre-processing of $16 \mathrm{~S}$ rRNA sequence data were performed on resources provided by the Swedish National Infrastructure for Computing (SNIC) through the Uppsala Multidisciplinary Center for Advanced Computational Science (UPPMAX). This study was supported by the Swedish Research Council, the Novo Nordisk Foundation, Torsten Söderberg's Foundation, Swedish Diabetes Foundation, Swedish Heart Lung Foundation, Göran Gustafsson's Foundation, IngaBritt och Arne Lundbergs Foundation, Knut and Alice Wallenberg Foundation, the Swedish Foundation for Strategic Research, the regional agreement on medical training and clinical research (ALF) between Region Västra Götaland and Sahlgrenska University Hospital and Science Foundation Ireland (SFI) ref 12/YI/B2480. FB is a recipient of an ERC Consolidator Grant (European Research Council, Consolidator grant 615362-METABASE). The financial support by the Deutsche Forschungsgemeinschaft and the Interdisciplinary Center fo Clinical Research Wuerzburg (Florian Seyfried, SE 2027/1-1 and IZKF-Z-3/44) is gratefully acknowledged.

\section{References}

Anderson MJ. (2001). A new method for non-parametric multivariate analysis of variance. Austral Ecol 26: $32-46$.

Arora T, Velagapudi V, Pournaras DJ, Welbourn R, le Roux CW, Oresic M et al. (2015). Roux-en-Y gastric bypass surgery induces early plasma metabolomic and lipidomic alterations in humans associated with diabetes remission. PLoS One 10: e0126401.

Backhed F, Ding H, Wang T, Hooper LV, Koh GY, Nagy A et al. (2004). The gut microbiota as an environmental factor that regulates fat storage. Proc Natl Acad Sci USA 101: 15718-15723.

Brethauer SA, Aminian A, Romero-Talamas H, Batayyah E, Mackey J, Kennedy L et al. (2013). Can diabetes be surgically cured? Long-term metabolic effects of bariatric surgery in obese patients with type 2 diabetes mellitus. Ann Surg 258: 628-637.

Caesar R, Tremaroli V, Kovatcheva-Datchary P, Cani PD, Backhed F. (2015). Crosstalk between gut microbiota and dietary lipids aggravates WAT inflammation through TLR signaling. Cell Metab 22: 658-668.

Caporaso JG, Kuczynski J, Stombaugh J, Bittinger K, Bushman FD, Costello EK et al. (2010a). QIIME allows analysis of high-throughput community sequencing data. Nat Methods 7: 335-336.

Caporaso JG, Bittinger K, Bushman FD, DeSantis TZ, Andersen GL, Knight R. (2010b). PyNAST: a flexible tool for aligning sequences to a template alignment. Bioinformatics 26: 266-267.
Carlsson LM, Peltonen M, Ahlin S, Anveden A, Bouchard C, Carlsson B et al. (2012). Bariatric surgery and prevention of type 2 diabetes in Swedish obese subjects. N Engl J Med 367: 695-704.

Cohen R, Caravatto PP, Correa JL, Noujaim P, Petry TZ, Salles JE et al. (2012). Glycemic control after stomachsparing duodenal-jejunal bypass surgery in diabetic patients with low body mass index. Surg Obes Relat Dis 8: $375-380$.

DeSantis TZ, Hugenholtz P, Larsen N, Rojas M, Brodie EL, Keller K et al. (2006). Greengenes, a chimerachecked $16 \mathrm{~S}$ rRNA gene database and workbench compatible with ARB. Appl Environ Microbiol 72: 5069-5072.

Edgar RC. (2010). Search and clustering orders of magnitude faster than BLAST. Bioinformatics 26: $2460-2461$.

Fak F, Tremaroli V, Bergstrom G, Backhed F. (2015). Oral microbiota in patients with atherosclerosis. Atherosclerosis 243: 573-578.

Gavin TP, Sloan 3rd RC, Lukosius EZ, Reed MA, Pender JR, Boghossian V et al. (2011). Duodenal-jejunal bypass surgery does not increase skeletal muscle insulin signal transduction or glucose disposal in Goto-Kakizaki type 2 diabetic rats. Obes Surg 21: 231-237.

Graessler J, Bornstein TD, Goel D, Bhalla VP, Lohmann T, Wolf T et al. (2013a). Lipidomic profiling before and after Roux-en-Y gastric bypass in obese patients with diabetes. Pharmacogenomics J 14: 201-207.

Graessler J, Qin Y, Zhong H, Zhang J, Licinio J, Wong ML et al. (2013b). Metagenomic sequencing of the human gut microbiome before and after bariatric surgery in obese patients with type 2 diabetes: correlation with inflammatory and metabolic parameters. Pharmacogenomics J 13: 514-522.

Greenwood MR, Maggio CA, Koopmans HS, Sclafani A. (1982). Zucker fafa rats maintain their obese body composition ten months after jejunoileal bypass surgery. Int J Obes 6: 513-525.

Haas BJ, Gevers D, Earl AM, Feldgarden M, Ward DV, Giannoukos G et al. (2011). Chimeric 16S rRNA sequence formation and detection in Sanger and 454pyrosequenced PCR amplicons. Genome Res 21: 494-504.

Hamady M, Walker JJ, Harris JK, Gold NJ, Knight R. (2008). Error-correcting barcoded primers for pyrosequencing hundreds of samples in multiplex. Nat Methods 5: 235-237.

Ionescu E, Sauter JF, Jeanrenaud B. (1985). Abnormal oral glucose tolerance in genetically obese (fa/fa) rats. Am J Physiol 248: E500-E506.

Isbell JM, Tamboli RA, Hansen EN, Saliba J, Dunn JP, Phillips SE et al. (2010). The importance of caloric restriction in the early improvements in insulin sensitivity after Roux-en-Y gastric bypass surgery. Diabetes Care 33: 1438-1442.

Karlsson FH, Tremaroli V, Nookaew I, Bergstrom G, Behre CJ, Fagerberg B et al. (2013). Gut metagenome in European women with normal, impaired and diabetic glucose control. Nature 498: 99-103.

Kindel TL, Yoder SM, Seeley RJ, D'Alessio DA, Tso P. (2009). Duodenal-jejunal exclusion improves glucose tolerance in the diabetic, Goto-Kakizaki rat by a GLP-1 receptor-mediated mechanism. J Gastrointest Surg 13: 1762-1772. 
Klein S, Fabbrini E, Patterson BW, Polonsky KS, Schiavon CA, Correa JL et al. (2012). Moderate effect of duodenal-jejunal bypass surgery on glucose homeostasis in patients with type 2 diabetes. Obesity 20: 1266-1272.

Kong LC, Tap J, Aron-Wisnewsky J, Pelloux V, Basdevant A, Bouillot JL et al. (2013). Gut microbiota after gastric bypass in human obesity: increased richness and associations of bacterial genera with adipose tissue genes. Am J Clin Nutr 98: 16-24.

Kozich JJ, Westcott SL, Baxter NT, Highlander SK, Schloss PD. (2013). Development of a dual-index sequencing strategy and curation pipeline for analyzing amplicon sequence data on the MiSeq Illumina sequencing platform. Appl Environ Microbiol 79: 5112-5120.

Larsen N, Vogensen FK, van den Berg FWJ, Nielsen DS, Andreasen AS, Pedersen BK et al. (2010). Gut microbiota in human adults with type 2 diabetes differs from non-diabetic adults. PLoS One 5: e9085.

Le KA, Li Y, Xu X, Yang W, Liu T, Zhao X et al. (2012). Alterations in fecal Lactobacillus and Bifidobacterium species in type 2 diabetic patients in Southern China population. Front Physiol 3: 496.

le Roux CW, Aylwin SJ, Batterham RL, Borg CM, Coyle F, Prasad V et al. (2006). Gut hormone profiles following bariatric surgery favor an anorectic state, facilitate weight loss, and improve metabolic parameters. Ann Surg 243: 108-114.

Lees H, Swann J, Poucher SM, Nicholson JK, Holmes E, Wilson ID et al. (2014). Age and microenvironment outweigh genetic influence on the Zucker rat microbiome. PLoS One 9: e100916.

Li B, Lu Y, Srikant CB, Gao ZH, Liu JL. (2013). Intestinal adaptation and Reg gene expression induced by antidiabetic duodenal-jejunal bypass surgery in Zucker fatty rats. Am J Physiol Gastrointest Liver Physiol 304: G635-G645.

Li JV, Ashrafian H, Bueter M, Kinross J, Sands C, le Roux CW et al. (2011). Metabolic surgery profoundly influences gut microbial-host metabolic cross-talk. Gut 60: $1214-1223$.

Lifante JC, Milone L, Korner J, Kopsombut G, Sebastian M, Inabnet WB 3rd. (2012). Sleeve gastrectomy improves glucose homeostasis in Zucker diabetic fatty rats. Obes Surg 22: 1110-1116.

Liou AP, Paziuk M, Luevano JM Jr, Machineni S, Turnbaugh PJ, Kaplan LM. (2013). Conserved shifts in the gut microbiota due to gastric bypass reduce host weight and adiposity. Sci Transl Med 5: 178 ra141.

Lozupone C, Knight R. (2005). UniFrac: a new phylogenetic method for comparing microbial communities. Appl Environ Microbiol 71: 8228-8235.

Marlene R, Simone D, Berit H, Jutta Z, Eva A, Helmut B et al. (2013). Abundance and diversity of microbiota in type 2 diabetes and obesity. J Diabetes Metab 4: 253.

Mutch DM, Fuhrmann JC, Rein D, Wiemer JC, Bouillot JL, Poitou C et al. (2009). Metabolite profiling identifies candidate markers reflecting the clinical adaptations associated with Roux-en-Y gastric bypass surgery. PLoS One 4: e7905.

Nguyen NQ, Debreceni TL, Bambrick JE, Chia B, Deane AM, Wittert G et al. (2014). Upregulation of intestinal glucose transporters after Roux-en-Y gastric bypass to prevent carbohydrate malabsorption. Obesity 22: 2164-2171.
Patel RT, Shukla AP, Ahn SM, Moreira M, Rubino F. (2013). Surgical control of obesity and diabetes: the role of intestinal vs. gastric mechanisms in the regulation of body weight and glucose homeostasis. Obesity 22: 159-169.

Qin J, Li Y, Cai Z, Li S, Zhu J, Zhang F et al. (2012). A metagenome-wide association study of gut microbiota in type 2 diabetes. Nature 490: 55-60.

Rubino F, Forgione A, Cummings DE, Vix M, Gnuli D, Mingrone G et al. (2006). The mechanism of diabetes control after gastrointestinal bypass surgery reveals a role of the proximal small intestine in the pathophysiology of type 2 diabetes. Ann Surg 244: 741-749.

Rubino F, Zizzari P, Tomasetto C, Bluet-Pajot MT, Forgione A, Vix M et al. (2005). The role of the small bowel in the regulation of circulating ghrelin levels and food intake in the obese Zucker rat. Endocrinology 146: 1745-1751.

Saeidi N, Meoli L, Nestoridi E, Gupta NK, Kvas S, Kucharczyk J et al. (2013). Reprogramming of intestinal glucose metabolism and glycemic control in rats after gastric bypass. Science 341: 406-410.

Salonen A, Nikkila J, Jalanka-Tuovinen J, Immonen O, Rajilic-Stojanovic M, Kekkonen RA et al. (2010). Comparative analysis of fecal DNA extraction methods with phylogenetic microarray: effective recovery of bacterial and archaeal DNA using mechanical cell lysis. J Microbiol Methods 81: 127-134.

Seyfried F, Bueter M, Spliethoff K, Miras AD, Abegg K, Lutz TA et al. (2014). Roux-en Y gastric bypass is superior to duodeno-jejunal bypass in improving glycaemic control in Zucker diabetic fatty rats. Obes Surg 24: 1888-1895.

Simonen M, Dali-Youcef N, Kaminska D, Venesmaa S, Kakela P, Paakkonen M et al. (2012). Conjugated bile acids associate with altered rates of glucose and lipid oxidation after Roux-en-Y gastric bypass. Obes Surg 22: 1473-1480.

Sjostrom L, Peltonen M, Jacobson P, Ahlin S, AnderssonAssarsson J, Anveden A et al. (2014). Association of bariatric surgery with long-term remission of type 2 diabetes and with microvascular and macrovascular complications. JAMA 311: 2297-2304.

Speck M, Cho YM, Asadi A, Rubino F, Kieffer TJ. (2011). Duodenal-jejunal bypass protects GK rats from \{beta\}-cell loss and aggravation of hyperglycemia and increases enteroendocrine cells coexpressing GIP and GLP-1. Am J Physiol Endocrinol Metab 300: E923-E932.

Tremaroli V, Karlsson F, Werling M, Stahlman M, Kovatcheva-Datchary P, Olbers T et al. (2015). Rouxen-Y gastric bypass and vertical banded gastroplasty induce long-term changes on the human gut microbiome contributing to fat mass regulation. Cell Metab 22: 228-238.

Vrieze A, Van Nood E, Holleman F, Salojärvi J, Kootte RS, Bartelsman JFWM et al. (2012). Transfer of intestinal microbiota from lean donors increases insulin sensitivity in individuals with metabolic syndrome. Gastroenterology 143: 913-916.e7.

Wolff BS, Meirelles K, Meng Q, Pan M, Cooney RN. (2009). Roux-en-Y gastric bypass alters small intestine glutamine transport in the obese Zucker rat. Am J Physiol Gastrointest Liver Physiol 297: G594-G601. 
Xu Y, Ohinata K, Meguid MM, Marx W, Tada T, Chen C et al. (2002). Gastric bypass model in the obese rat to study metabolic mechanisms of weight loss. J Surg Res 107: 56-63.

Yakovlieva M, Tacheva T, Mihaylova S, Tropcheva R, Trifonova K. (2015). Influence of Lactobacillus brevis 15 and Lactobacillus plantarum 13 on blood glucose and body weight in rats after high-fructose diet. Benef Microbes 6: 505-512.

Zhang H, DiBaise JK, Zuccolo A, Kudrna D, Braidotti M, $\mathrm{Yu} \mathrm{Y}$ et al. (2009). Human gut microbiota in obesity and after gastric bypass. Proc Natl Acad Sci USA 106: 2365-2370. (c) (1) (2) (2) This work is licensed under a Creative Commons Attribution-NonCommercialShareAlike 4.0 International License. The images or other third party material in this article are included in the article's Creative Commons license, unless indicated otherwise in the credit line; if the material is not included under the Creative Commons license, users will need to obtain permission from the license holder to reproduce the material. To view a copy of this license, visit http://creativecommons.org/ licenses/by-nc-sa/4.0/

(C) The Author(s) 2017

Supplementary Information accompanies this paper on The ISME Journal website (http://www.nature.com/ismej) 